\title{
Non-perturbatively Renormalized Light Quark Masses with Two Dynamical Fermions
}

\section{Bećirević ${ }^{a}$, B. Blossier ${ }^{a}$, Ph. Boucaud ${ }^{a}$, V. Giménez ${ }^{b}$, V. Lubicz ${ }^{c, d}$, F. Mescia $^{c, e}$,} S. Simula ${ }^{d}$, and C. Tarantino ${ }^{* c, d}$

${ }^{a}$ Univ Paris Sud, ${ }^{b}$ Univ València and IFIC, ${ }^{c}$ Univ Roma Tre, ${ }^{d}$ INFN (Roma III), ${ }^{e}$ INFN (LNF)

*tarantinodfis.uniroma3.it

We present the results of a partially quenched lattice QCD calculation of light quark masses with $N_{f}=2$ degenerate dynamical flavors. Numerical simulations are carried out using the plaquette gauge action and the Wilson quark action at $\beta=5.8\left(a^{-1} \simeq 3.2 \mathrm{GeV}\right)$. The spatial extension of the $24^{3} \times 48$ lattice is about $1.5 \mathrm{fm}$. Configurations have been generated at four values of the sea quark masses, for which the ratio of pseudoscalar over vector meson masses is in the range $M_{P} / M_{V} \simeq 0.60 \div 0.75$. An important feature of the present study is the use of non-perturbative renormalization, performed with the RI-MOM method. The effects of dynamical sea quarks in the determination of light quark masses have been investigated by performing a quenched calculation on a similar lattice. Our results for the average up-down and strange quark masses are $m_{u d}^{\overline{\mathrm{MS}}}(2 \mathrm{GeV})=4.3(4)\left({ }_{-0.4}^{+1.1}\right) \mathrm{MeV}$ and $m_{s}^{\overline{\mathrm{MS}}}(2 \mathrm{GeV})=101(8)\left({ }_{-9}^{+25}\right) \mathrm{MeV}$. These values are larger than those obtained by evaluating the quark mass renormalization constants with one-loop (boosted) perturbation theory. Our results for the light quark masses are compatible with those obtained in the quenched simulation. No significant sea quark effects are seen, at the values of sea quark masses used in the present study.

XXIIIrd International Symposium on Lattice Field Theory

25-30 July 2005

Trinity College, Dublin, Ireland

\footnotetext{
* Speaker.
} 


\section{Introduction}

In recent years, lattice QCD calculations of light quark masses have significantly improved. Within the quenched approximation, i.e. ignoring quark vacuum polarization effects, the accuracy reached by these calculations has become better than $10 \%$, and the quenching error in these studies represents the main source of uncertainty. Lattice QCD calculations of light quark masses with both $N_{f}=2$ and $N_{f}=3$ dynamical quarks have been also performed [1]-[5]. The results seem to indicate that the inclusion of sea quarks effects decreases the quark mass estimates. The accuracy of partially quenched simulations, however, is still limited by several systematic uncertainties, which deserve to be further investigated. In particular, in all studies with dynamical fermions performed so far, with the exception of Refs. [4, 5], renormalization has been implemented by using one-loop boosted perturbation theory (BPT) [6].

The aim of the present study is to explore sea quark effects in the determination of light quark masses, using a non-perturbative renormalization technique. To this purpose, we have performed a lattice QCD simulation with $N_{f}=2$ dynamical quarks and compared the results with those obtained in a quenched simulation, at similar values of the lattice scale, lattice volume and quark masses. In both cases, the quark mass renormalization constants (RCs) have been computed by implementing the RI-MOM non-perturbative method of Ref. [7].

Numerical simulations are carried out with the LL-SSOR [8] preconditioned Hybrid Monte Carlo (HMC) [9], using the plaquette gauge action and the Wilson quark action at $\beta=5.8\left(a^{-1}=\right.$ $3.2(1) \mathrm{GeV})$. The spatial extension of the $24^{3} \times 48$ lattice is $L \simeq 1.5 \mathrm{fm}$. Gauge configurations have been generated at four values of the sea quark masses $\left(\kappa_{s}=\{0.1535,0.1538,0.1540,0.1541\}\right)$, for which the ratio of pseudoscalar over vector meson masses is in the range $M_{P} / M_{V} \simeq 0.60 \div 0.75^{1}$.

Our final results for the average up-down and strange quark masses are

$$
m_{u d}^{\overline{\mathrm{MS}}}(2 \mathrm{GeV})=4.3(4)\left({ }_{-0.4}^{+1.1}\right) \mathrm{MeV}, m_{s}^{\overline{\mathrm{MS}}}(2 \mathrm{GeV})=101(8)\left({ }_{-9}^{+25}\right) \mathrm{MeV}\left(N_{f}=2, \mathrm{RI}-\mathrm{MOM}\right)(1.1)
$$

where the second is the systematic error dominated by the difference between the results obtained with the vector or the axial-vector definitions of the bare quark masses. These results lie above the averages of the other partially quenched studies. We will see in the following that the main reason for that is the use of non-perturbative renormalization. The results of Eq. (1.1) will be also compared with the estimates of quark masses obtained in the quenched simulation. Within our statistical and systematic accuracy, and with the values of sea quark masses used in the present study, we do not observe any significant effect of dynamical quarks.

\section{Lattice spacing}

In order to study the light meson spectrum we have calculated two-point pseudoscalar and vector correlation functions, with both degenerate and non-degenerate valence quarks and with valence quark masses equal or different to the sea quark mass.

Lattice spacing and masses of the physical light mesons have been then computed by studying the dependence of pseudoscalar and vector meson masses on valence and sea quark masses. This

\footnotetext{
${ }^{1}$ For further simulation details we address the reader to Ref. [10].
} 
study can be conveniently done by investigating directly the dependence of vector meson masses on the pseudoscalar ones, with either degenerate or non-degenerate sea and valence quarks. The dependence on the valence quark mass is found to be linear, while the dependence on the sea quark mass is too weak to be determined in the range of simulated pseudoscalar masses $((580 \div$ 840) MeV).

The value of the inverse lattice spacing has been obtained, following the method of "lattice physical planes" [11], setting the ratio $M_{K} / M_{K}^{*}$ equal to its experimental value. We obtain the estimate $a^{-1}=3.2(1) \mathrm{GeV}$.

\section{Renormalization Constants}

The determination of the RCs has been performed non-perturbatively by using the RI-MOM method [7], which is based on the numerical evaluation in momentum space of correlation functions of the relevant operators between external quark states, in a fixed (Landau) gauge. We have computed the RCs of the complete set of bilinear quark operators, $\mathscr{O}_{\Gamma}=\bar{q} \Gamma q$, with $\Gamma=S, P, V, A, T$ standing respectively for $I, \gamma_{5}, \gamma_{\mu}, \gamma_{\mu} \gamma_{5}$, and $\sigma_{\mu \nu}$. We also present the result for the RC of the quark field, $Z_{q}$.

For the scale dependent bilinear operators, namely $\mathscr{O}=S, P, T$, we have computed the renormalization group invariant combinations

$$
Z_{\mathscr{O}}\left(\mu_{0}\right)=C_{\mathscr{O}}\left(\mu_{0}\right) Z_{\mathscr{O}}^{R G I}=C_{\mathscr{O}}\left(\mu_{0}\right)\left(Z_{\mathscr{O}}(\mu) / C_{\mathscr{O}}(\mu)\right),
$$

where the evolution functions $C_{\mathscr{O}}(\mu)$ are introduced in order to explicitly cancel, at a given order in the renormalization group improved perturbation theory, the scale dependence of the RCs. In the RI-MOM scheme, these functions are known at the $\mathrm{N}^{3} \mathrm{LO}$ for $Z_{S}$ and $Z_{P}$ [12] and at $\mathrm{N}^{2} \mathrm{LO}$ for $Z_{T}$ [13]. In the particular case of the scalar density RC, $Z_{S}$, we observe a smooth linear dependence on the initial renormalization scale $(a \mu)^{2}$, which is due to discretization or higher order perturbative effects. In order to correct for these effects, our estimates of the RCs have been obtained by linearly extrapolating to $(a \mu)^{2}=0$.

The renormalization condition which defines the RI-MOM renormalization scheme, must be implemented in the chiral limit, in order to ensure the mass independence of this scheme. Since the non-perturbative determinations of the RCs are obtained at non vanishing values of the sea and valence quark masses, an extrapolation of the results to the chiral limit, which results to be linear in both sea and valence masses, has been also performed.

An important comparison to be performed is the one between the non-perturbative results and the predictions of one-loop perturbation theory ${ }^{2}$. The reason is that the perturbative approach has been the only one considered so far in the determinations of the quark mass RCs in partially quenched lattice calculations, with the exception of Refs. [甘, 5]. In the present study we have considered two versions of boosted perturbation theory (BPT), the so called naive and tadpole improved BPT [15]. The predictions for the RCs of bilinear quark operators obtained by using the non-perturbative RI-MOM method, and one-loop naive and tadpole improved BPT are collected in Table 1 .

\footnotetext{
${ }^{2}$ Recently, an accurate study of the bilinear operator RCs has been performed to four loops by using the Stochastic Perturbation Theory [14].
} 


\begin{tabular}{||l||c|c|c||}
\hline \hline & RI-MOM & N-BPT & TI-BPT \\
\hline \hline$Z_{V}$ & $0.66(2)$ & 0.70 & 0.65 \\
\hline$Z_{A}$ & $0.76(1)$ & 0.77 & 0.73 \\
\hline$Z_{S}$ & $0.67(3)$ & 0.75 & 0.71 \\
\hline$Z_{P}$ & $0.50(2)$ & 0.61 & 0.55 \\
\hline$Z_{T}$ & $0.74(4)$ & 0.75 & 0.71 \\
\hline$Z_{q}$ & $0.76(1)$ & 0.75 & 0.72 \\
\hline \hline
\end{tabular}

Table 1: Comparison of RCs at the scale $\mu=1 /$ a, obtained with both perturbative and non-perturbative methods: RI-MOM, Naive BPT and Tadpole Improved BPT.

We note in particular that, in the case of the axial-vector WI definition of the quark mass, the non-perturbative RI-MOM value of the quark mass $\mathrm{RC}, Z_{m}^{A W I}=Z_{A} / Z_{P}$, is larger by approximately $15 \%$ than its estimate obtained from tadpole improved BPT. In the case of the vector WI definition, $Z_{m}^{V W I}=1 / Z_{S}$, the non-perturbative value is instead larger by approximately $6 \%$ than the perturbative one. These differences propagate directly in the estimates of the renormalized quark masses. We anticipate that numerically they can completely explain the differences between our non-perturbatively renormalized results and the partially quenched averages obtained using BPT.

\section{Light quark masses}

In order to determine the values of the light quark masses we consider both vector and axialvector WI definitions of these quantities. The vector Ward identity (VWI) relates the bare quark mass to the Wilson hopping parameter and the quark mass RC to the one of the scalar density. The axial-vector Ward identity (AWI), instead, is based on the PCAC relation and relates the quark mass $\mathrm{RC}$ to those of the axial and pseudoscalar operators. An important observation is in order here. The bare quark mass depends on the value of the sea hopping parameter. In the AWI definition $a m_{q}^{A W I}=\left\langle\sum_{\vec{x}} \partial_{0} A_{0}(x) P^{\dagger}(0)\right\rangle /\left(2\left\langle\sum_{\vec{x}} P(x) P^{\dagger}(0)\right\rangle\right)$, this dependence is provided by the ratio of the 2-point correlation functions. In the VWI definition $a m_{q}^{V W I}=\left(1 / \kappa_{q}-\kappa_{c r}\left(\kappa_{s}\right)\right) / 2$, instead, the dependence on the sea quark mass is given by the value of the critical hopping parameter.

In order to get the physical values of the light quark masses, we have studied the dependence of pseudoscalar meson masses on valence and sea quark masses. Inspired by partially quenched ChPT [16, 17], and neglecting chiral logs, we have attempted a fit of pseudoscalar meson masses to the form

$$
\left[a M_{P}\left(\kappa_{s}, \kappa_{v_{1}}, \kappa_{v_{2}}\right)\right]^{2}=R_{1} a m_{v}\left[1+R_{2} a m_{s}+R_{3} a m_{v}\right], \quad \text { with } a m_{v}=\frac{a m_{v 1}+a m_{v 2}}{2} .
$$

Discretization effects entering either pseudoscalar meson masses either quark masses or both, affect this fit. As a consequence the pseudoscalar meson mass does not vanish when the AWI quark mass goes to the chiral limit and an additional constant term, $a R_{0}\left(\kappa_{s}\right)$, appears in fit (4.1). When fit (4.1) is performed in terms of VWI quark masses, instead, the discretization contribution is implicitly reabsorbed in the fitted values of the critical hopping parameters $\kappa_{c r}\left(\kappa_{s}\right)$. 

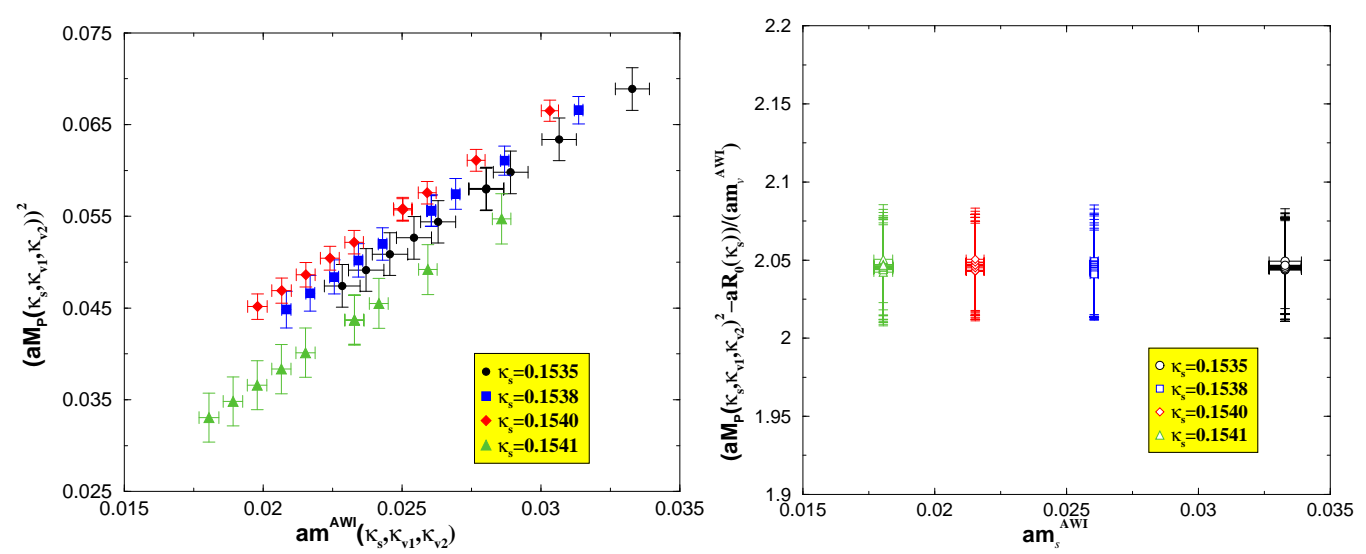

Figure 1: Dependence of pseudoscalar meson masses on valence quark masses am ${ }_{v}^{A W I}$ (left) and of the ratios $\left(a M_{P}\right)^{2} / a m_{v}^{A W I}$ on sea quark masses am $m_{s}^{A W I}$.

The dependence of squared pseudoscalar meson masses on AWI valence and sea quark masses is illustrated in Fig. 1 (the VWI case presents a similar behavior). From the left plot we observe a clear linear dependence on the valence quark mass. On the other hand, once this dependence is factorized out, the right plot shows a completely flat behaviour in the sea quark mass. Our final results are obtained by setting equal to zero the quadratic coefficients in fit (4.1).

We now present the partially quenched results of the average up-down $\left(m_{u d}\right)$ and strange $\left(m_{s}\right)$ quark masses. They have been obtained by substituting on the 1.h.s. of Eq. (4.1) the experimental values of pion and kaon masses and using the estimate of the lattice spacing $a^{-1}=3.2(1) \mathrm{GeV}$. The values of the bare quark masses are then converted to the renormalized quark masses in the RI-MOM scheme at the scale $\mu_{0}=1 / a$, by using the RCs computed non-perturbatively with the RI-MOM method and given in Table 1. Finally, the results are translated to the $\overline{\mathrm{MS}}$, at the conventional scale $\mu=2 \mathrm{GeV}$, by using renormalization group improved perturbation theory at 4-loop accuracy [12]. We find large differences $(\sim 15 \%)$ between the VWI and AWI determinations of quark masses, which have to be attributed to discretization effects. It has been shown in Refs. [1] 2] that the differences between the VWI and AWI determinations decrease when going to smaller lattice spacing, and vanish, as expected, in the continuum limit. In addition, they have found that, when working at fixed lattice spacing, discretization errors affecting the VWI determination of quark masses are significantly larger than those observed in the AWI case. Motivated by these findings, we quote as final results for the light quark masses those obtained from the AWI determination, including the difference between the VWI and AWI results in the systematic asymmetric error

$$
m_{u d}^{\overline{\mathrm{MS}}}(2 \mathrm{GeV})=4.3(4)\left({ }_{-0.4}^{+1.1}\right) \mathrm{MeV}, m_{s}^{\overline{\mathrm{MS}}}(2 \mathrm{GeV})=101(8)\left({ }_{-9}^{+25}\right) \mathrm{MeV} \quad\left(N_{f}=2, \mathrm{RI}-\mathrm{MOM}\right)(4.2)
$$

The second (systematic) error also includes differences between the quoted central values and the results we would obtain by fixing the lattice spacing from the Sommer scale $r_{0}$ [18] $\left(a_{r_{0}}^{-1}=\right.$ $3.4(2) \mathrm{GeV}$ ), or by using hadronic Ward Identities to determinate the RCs [19]. Had we used oneloop tadpole improved BPT, instead of the non-perturbative RI-MOM renormalization method, we would have obtained

$$
m_{u d}^{\overline{\mathrm{MS}}}(2 \mathrm{GeV})=3.7(3)\left({ }_{-0}^{+1.1}\right) \mathrm{MeV}, m_{s}^{\overline{\mathrm{MS}}}(2 \mathrm{GeV})=88(7)\left({ }_{-0}^{+25}\right) \mathrm{MeV}\left(N_{f}=2, \mathrm{TI}-\mathrm{BPT}\right)(4.3)
$$


As anticipated in Sec. 3, the non-perturbative estimates of the quark mass RCs tend to increase the final predictions for the quark masses, by a non negligible amount, thus explaining the differences with previous BPT partially quenched results.

A final remark concerns the results obtained from the quenched simulation performed at $V=$ $24^{3} \times 56, \beta=6.2\left(a^{-1}=2.98(7) \mathrm{GeV}\right)$

$$
m_{u d}^{\overline{\mathrm{MS}}}(2 \mathrm{GeV})=4.6(2)\left({ }_{-0}^{+0.5}\right) \mathrm{MeV}, m_{s}^{\overline{\mathrm{MS}}}(2 \mathrm{GeV})=106(2)\left({ }_{-0}^{+12}\right) \mathrm{MeV}\left(N_{f}=0, \mathrm{RI}-\mathrm{MOM}\right)(4.4)
$$

No significant differences with the partially quenched results in Eq. (4.2) are observed within the statistical and systematic uncertainties. We believe that a better indication of sea quark effects in the lattice determinations of light quark masses could be achieved by performing simulations with higher statistics and using more widespread values of sea quark masses.

\section{Acknowledgements}

It is a pleasure to thank Guido Martinelli for useful discussions on the subject of this paper.

\section{References}

[1] A. Ali Khan et al. [CP-PACS Collaboration], Phys. Rev. D 65 (2002) 054505 [Erratum-ibid. D 67 (2003) 059901] [hep-lat/0105015].

[2] S. Aoki et al. [JLQCD Collaboration], Phys. Rev. D 68 (2003) 054502 [hep-lat/0212039].

[3] T. Ishikawa et al. [CP-PACS Collaboration], Nucl. Phys. Proc. Suppl. 140 (2005) 225 [hep-lat/0409124].

[4] M. Gockeler et al. [QCDSF Collaboration], hep-ph/0409312.

[5] M. Della Morte et al. [ALPHA Collaboration], hep-lat/0507035.

[6] G. P. Lepage and P. B. Mackenzie, Phys. Rev. D 48 (1993) 2250 [hep-lat/9209022].

[7] G. Martinelli et al., Nucl. Phys. B 445 (1995) 81 [hep-lat/9411010].

[8] S. Fischer et al., Comput. Phys. Commun. 98 (1996) 20 [hep-lat/9602019].

[9] S. Duane et al., Phys. Lett. B 195 (1987) 216.

S. A. Gottlieb et al., Phys. Rev. D 35, 2531 (1987).

[10] D. Becirevic et al., in phase of publication.

[11] C. R. Allton et al., Nucl. Phys. B 489 (1997) 427 [hep-lat/9611021].

[12] K. G. Chetyrkin and A. Retey, Nucl. Phys. B 583 (2000) 3 [hep-ph/9910332].

[13] J. A. Gracey, Nucl. Phys. B 662 (2003) 247 [hep-ph/0304113].

[14] F. Di Renzo et al., these proceedings.

[15] M. Crisafulli et al., Eur. Phys. J. C 4 (1998) 145 [hep-lat/9707025].

[16] C. W. Bernard and M. F. L. Golterman, Phys. Rev. D 49 (1994) 486 [hep-lat/9306005].

[17] S. R. Sharpe and N. Shoresh, Phys. Rev. D 62 (2000) 094503 [hep-lat/0006017].

[18] R. Sommer, Nucl. Phys. B 411 (1994) 839 [hep-lat/9310022].

[19] M. Bochicchio et al., Nucl. Phys. B 262 (1985) 331. 